\title{
Adaptive Fuzzy Tracking Control for a Permanent Magnet Synchronous Motor via Backstepping Approach
}

\author{
Jinpeng Yu, ${ }^{1,2}$ Junwei Gao, ${ }^{1,3}$ Yumei $\mathrm{Ma}^{1}{ }^{1}$ and Haisheng $\mathbf{Y u}^{1,2}$ \\ ${ }^{1}$ Institute of Complexity Science, Qingdao University, Qingdao 266071, China \\ 2 Shan Dong Province Key Laboratory of Industrial Control Technique, Qingdao University, \\ Qingdao 266071, China \\ ${ }^{3}$ State Key Laboratory of Rail Traffic Control and Safety, Beijing Jiaotong University, \\ Beijing 100044, China
}

Correspondence should be addressed to Jinpeng Yu, yjp1109@hotmail.com

Received 16 July 2009; Accepted 30 September 2009

Academic Editor: José Balthazar

Copyright (C) 2010 Jinpeng Yu et al. This is an open access article distributed under the Creative Commons Attribution License, which permits unrestricted use, distribution, and reproduction in any medium, provided the original work is properly cited.

The speed tracking control problem of permanent magnet synchronous motors with parameter uncertainties and load torque disturbance is addressed. Fuzzy logic systems are used to approximate nonlinearities, and an adaptive backstepping technique is employed to construct controllers. The proposed controller guarantees the tracking error convergence to a small neighborhood of the origin and achieves the good tracking performance. Simulation results clearly show that the proposed control scheme can track the position reference signal generated by a reference model successfully under parameter uncertainties and load torque disturbance without singularity and overparameterization.

\section{Introduction}

Permanent magnet synchronous motors (PMSMs) are of great interest for industrial applications requiring dynamic performance due to their high speed, high efficiency, high power density, and large torque to inertia ratio. Then it is still a challenging problem to control PMSM to get the perfect dynamic performance because the motor dynamic model of PMSM is nonlinear and multivariable, the model parameters such as the stator resistance and the friction coefficient are also not be exactly known. The control of PMSM drivers has recently received wide attention and become an active research area. Some advanced control techniques, such as sliding mode control [1, 2], feedback linearization control [3], adaptive control $[2,4]$, backstepping principles [5-7], and Fuzzy logic control [8-10], are used to the problems of speed or position control of PMSMs. 
Table 1: The denotation of the PMSM parameters.

\begin{tabular}{lccc}
\hline Parameter & Denotation & Parameter & Denotation \\
\hline$\omega$ & The rotor angular velocity & $R_{S}$ & The stator resistance \\
$i_{d}$ & The $d$ axis current & $i_{q}$ & The $q$ axis current \\
$L_{d}$ & The $d$ axis stator inductor & $L_{q}$ & The $q$ axis stator inductor \\
$u_{d}$ & The $d$ axis voltage & $u_{q}$ & The $q$ axis voltage \\
$n_{p}$ & The pole pair & $J$ & The rotor moment of inertia \\
$T$ & The electromagnetism torque & $T_{L}$ & The load torque \\
$B$ & The viscous friction coefficient & $\Phi$ & The magnet flux linkage of inertia \\
\hline
\end{tabular}

Backstepping is a newly developed technique to control the nonlinear systems with parameter uncertainty, particularly those systems in which the uncertainty does not satisfy matching conditions. Though backstepping is successfully applied to the control of PMSM drivers recently, it usually makes the designed controllers' structure to be very complex.

Fuzzy logic control (FLC) has been found one of the most popular and conventional tools in functional approximations. An FLC $[11,12]$ has strong ability of handling uncertain information and can be easily used in the control of systems which is ill-defined or too complex to have a mathematical model. It provides an effective way to design control system that is one of important applications in the area of control engineering.

In this paper, an adaptive fuzzy control approach is proposed for speed tracking control of PMSM drive system via the backstepping technique. During the controller design process, fuzzy logic systems are employed to approximate the nonlinearities, the adaptive fuzzy controllers are constructed via backstepping. The designed fuzzy controller can track the reference signal quite well even the existence of the parameter uncertainties and load torque disturbance. Compared with the existing controller design schemes via backstepping, the proposed method is very simple and the proposed controller has a simple structure.

\section{Mathematical Model of the PMSM Drive System and Preliminaries}

In this section, some preparatory knowledge of a PMSM will be introduced. The following assumptions are made in the derivation of the mathematical model of a PMSM [13].

Assumption 2.1. Saturation and iron losses are neglected although it can be taken into account by parameter changes.

Assumption 2.2. The back emf is sinusoidal.

The model of a PMSM can be described in the well known $(d-q)$ frame through the Park transformation as follows. The stator $d, q$ equations in the rotor frame are expressed as follows [14]:

$$
\begin{gathered}
J \frac{d \omega}{d t}=T-T_{L}-B \omega=\frac{3}{2} n_{p}\left[\left(L_{d}-L_{q}\right) i_{d} i_{q}+\Phi i_{q}\right]-B \omega-T_{L}, \\
L_{d} \frac{d i_{d}}{d t}=-R_{s} i_{d}+n_{p} \omega L_{q} i_{q}+u_{d}, \\
L_{q} \frac{d i_{q}}{d t}=-R_{s} i_{q}-n_{p} \omega L_{d} i_{d}-n_{p} \omega \Phi+u_{q} .
\end{gathered}
$$

The denotation of the PMSM parameters is shown in Table 1. 
To simplify the previous method mode, the following notations are introduced:

$$
\begin{gathered}
x_{1}=\omega, \quad x_{2}=i_{q}, \quad x_{3}=i_{d}, \\
a_{1}=\frac{3 n_{p} \Phi}{2}, \quad a_{2}=\frac{3 n_{p}\left(L_{d}-L_{q}\right)}{2}, \\
b_{1}=-\frac{R_{s}}{L_{q}}, \quad b_{2}=-\frac{n_{p} L_{d}}{L_{q}}, \quad b_{3}=-\frac{n_{p} \Phi}{L_{q}}, \quad b_{4}=\frac{1}{L_{q}} \\
c_{1}=-\frac{R_{s}}{L_{d}}, \quad c_{2}=\frac{n_{p} L_{q}}{L_{d}}, \quad c_{3}=\frac{1}{L_{d}} .
\end{gathered}
$$

By using these notations, the dynamic model of a PMSM motor can be described by the following differential equations:

$$
\begin{aligned}
& \dot{x}_{1}=\frac{a_{1}}{J} x_{2}+\frac{a_{2}}{J} x_{2} x_{3}-\frac{B}{J} x_{1}-\frac{T_{L}}{J}, \\
& \dot{x}_{2}=b_{1} x_{2}+b_{2} x_{1} x_{3}+b_{3} x_{1}+b_{4} u_{q}, \\
& \dot{x}_{3}=c_{1} x_{3}+c_{2} x_{1} x_{2}+c_{3} u_{d} .
\end{aligned}
$$

The control objective is to design an adaptive fuzzy controller such that the state variable $x_{1}$ tracks the given reference signal $x_{d}$ and all signals of the resulting closed-loop system are uniformly ultimately bounded. In this paper, we adopt the singleton fuzzifier, product inference, and the center-defuzzifier to deduce the following fuzzy rules:

$$
R_{i} \text { : IF } x_{1} \text { is } F_{1}^{i} \text { and } \ldots \text { and } x_{n} \text { is } F_{n}^{i} \text { THEN } y \text { is } B^{i} \quad(i=1,2, \ldots, N) \text {, }
$$

where $x=\left[x_{1}, \ldots, x_{n}\right]^{T} \in R^{n}$, and $y \in R$ are the input and output of the fuzzy system, respectively, $F_{i}^{j}$ and $B^{i}$ are fuzzy sets in $R$. The fuzzy inference engine performs a mapping from fuzzy sets in $R^{n}$ to fuzzy set in $R$ based on the IF-THEN rules in the fuzzy rule base and the compositional rule of inference. The fuzzifier maps a crisp point $x=\left[x_{1}, \ldots, x_{n}\right]^{T} \in R^{n}$ into a fuzzy set $A_{x}$ in $R$. The defuzzifier maps a fuzzy set in $R$ to a crisp point in $R$. Since the strategy of singleton fuzzification, center-average defuzzification, and product inference is used, the output of the fuzzy system can be formulated as

$$
y(x)=\frac{\sum_{j=1}^{N} W_{j} \prod_{i=1}^{n} \mu_{F_{i}^{j}}\left(x_{i}\right)}{\sum_{j=1}^{N}\left[\prod_{i=1}^{n} \mu_{F_{i}^{j}}\left(x_{i}\right)\right]},
$$

where $W_{j}$ is the point at which fuzzy membership function $\mu_{B^{j}}\left(W_{j}\right)$ achieves its maximum value, and it is assumed that $\mu_{B^{j}}\left(W_{j}\right)=1$. Let $p_{j}(x)=\prod_{i=1}^{n} \mu_{F_{i}^{j}}\left(x_{i}\right) / \sum_{j=1}^{N}\left[\prod_{i=1}^{n} \mu_{F_{i}^{j}}\left(x_{i}\right)\right], S(x)=$ $\left[p_{1}(x), p_{2}(x), \ldots, p_{N}(x)\right]^{T}$ and $W=\left[W_{1}, \ldots, W_{N}\right]^{T}$, then the fuzzy logic system above can be 
rewritten as

$$
y(x)=W^{T} S(x)
$$

If all memberships are taken as Gussian functions, then the following lemma holds.

Lemma 2.3 (see [15]). Let $f(x)$ be a continuous function defined on a compact set $\Omega$. Then for any scalar $\varepsilon>0$, there exists a fuzzy logic system in the form (2.6) such that

$$
\sup _{x \in \Omega}|f(x)-y(x)| \leq \varepsilon .
$$

\section{Adaptive Fuzzy Controller with the Backstepping Technique}

For the system (2.3), the backstepping design procedure contains 3 steps. At each design step, a virtual control function $\alpha_{i}(i=1,2)$ will be constructed by using an appropriate Lyapunov function $V$. At the last step, a real controller is constructed to control the system. In the following, we will give the procedure of the backstepping design.

Step 1. For the reference signal $x_{d}$, define the tracking error variable as $z_{1}=x_{1}-x_{d}$. From the first subsystem of (2.3), the error dynamic system is given by $\dot{z}_{1}=\left(a_{1} / J\right) x_{2}+\left(a_{2} / J\right) x_{2} x_{3}-$ $(B / J) x_{1}-T_{L} / J-\dot{x}_{d}$.

Choose Lyapunov function candidate as $V_{1}=(J / 2) z_{1}^{2}$, then the time derivative of $V_{1}$ is given by

$$
\dot{V}_{1}=J z_{1} \dot{z}_{1}=z_{1}\left(a_{1} x_{2}+a_{2} x_{2} x_{3}-B x_{1}-T_{L}-J \dot{x}_{d}\right)
$$

As the parameters $B, T_{L}$, and $J$ are unknown, they cannot be used to construct the control signal. Thus, let $\widehat{B}, \widehat{T}_{L}$, and $\widehat{J}$ be their estimations of $B, T_{L}$, and $J$, respectively. The corresponding adaptation laws will be determined later. Now, construct the virtual control law $\alpha_{1}$ as

$$
\alpha_{1}\left(Z_{1}\right)=\frac{1}{a_{1}}\left(-k_{1} z_{1}+\widehat{B} x_{1}+\widehat{T}_{L}+\widehat{J} \dot{x}_{d}\right)
$$

where $k_{1}>0$ is a design parameter and $Z_{1}=\left[x_{1}, x_{d}, \dot{x}_{d}, \widehat{B}, \widehat{T}_{L}, \widehat{J}\right]^{T}$. Defining $z_{2}=x_{2}-\alpha_{1}$ and substituting (3.2) into (3.1) yield

$$
\dot{V}_{1}=-k_{1} z_{1}^{2}+a_{1} z_{1} z_{2}+a_{2} z_{1} x_{2} x_{3}+z_{1}(\widehat{B}-B) x_{1}+z_{1}\left(\widehat{T}_{L}-T_{L}\right)+z_{1}(\widehat{J}-J) \dot{x}_{d}
$$

Step 2. Differentiating $z_{2}$ and using the second subsystem of (2.3) give

$$
\dot{z}_{2}=\dot{x}_{2}-\dot{\alpha}_{1}=b_{1} x_{2}+b_{2} x_{1} x_{3}+b_{3} x_{1}+b_{4} u_{q}-\dot{\alpha}_{1} .
$$


Now, choose the Lyapunov function candidate as $V_{2}=V_{1}+(1 / 2) z_{2}^{2}$. Obviously, the time derivative of $V_{2}$ is given by

$$
\begin{aligned}
\dot{V}_{2} & =\dot{V}_{1}+z_{2} \dot{z}_{2} \\
& =-k_{1} z_{1}^{2}+a_{2} z_{1} x_{2} x_{3}+z_{1}(\widehat{B}-B) x_{1}+z_{1}\left(\widehat{T}_{L}-T_{L}\right)+z_{1}(\widehat{J}-J) \dot{x}_{d}+z_{2}\left(f_{2}+b_{4} u_{q}\right),
\end{aligned}
$$

where

$$
\begin{aligned}
\dot{\alpha}_{1} & =\frac{\partial \alpha_{1}}{\partial x_{1}} \dot{x}_{1}+\sum_{i=0}^{1} \frac{\partial \alpha_{1}}{\partial x_{d}^{(i)}} x_{d}^{(i+1)}+\frac{\partial \alpha_{1}}{\partial \widehat{B}} \dot{\widehat{B}}+\frac{\partial \alpha_{1}}{\partial \widehat{T}_{L}} \dot{\widehat{T}}_{L}+\frac{\partial \alpha_{1}}{\partial \widehat{J}} \dot{\hat{J}} \\
& =\frac{\partial \alpha_{2}}{\partial x_{1}}\left(\frac{a_{1}}{J} x_{2}+\frac{a_{2}}{J} x_{2} x_{3}-\frac{B}{J} x_{1}-\frac{T_{L}}{J}\right)+\sum_{i=0}^{1} \frac{\partial \alpha_{1}}{\partial x_{d}^{(i)}} x_{d}^{(i+1)}+\frac{\partial \alpha_{1}}{\partial \widehat{B}} \dot{\hat{B}}+\frac{\partial \alpha_{1}}{\partial \widehat{T}_{L}} \dot{\widehat{T}}_{L}+\frac{\partial \alpha_{1}}{\partial \widehat{J}}, \\
f_{2}\left(Z_{2}\right) & =a_{1} z_{1}+b_{1} x_{2}+b_{2} x_{1} x_{3}+b_{3} x_{1}-\dot{\alpha}_{1}, \\
Z_{2} & =\left[x_{1}, x_{2}, x_{3}, x_{d}, \dot{x}_{d}, \ddot{x}_{d}, \widehat{B}, \widehat{T}_{L}, \widehat{J}\right]^{T} .
\end{aligned}
$$

Apparently, there are two nonlinear terms in (3.5), that is, $a_{2} z_{1} x_{2} x_{3}$ and $f_{2}$, therewithal, $f_{2}$ contains the derivative of $\dot{\alpha}_{1}$. This will make the classical adaptive backstepping design become very complex and troubled, and the designed control law $u_{q}$ will have the complex structure. To avoid this trouble in design procedure and simplify the control signal structure, we will employ the fuzzy logic system to approximate the nonlinear function $f_{2}$. As shown later, the design procedure of $u_{q}$ becomes simple and $u_{q}$ has the simple structure. According to Lemma 2.3, for any given $\varepsilon_{2}>0$, there exists a fuzzy logic system $W_{2}^{T} S_{2}\left(Z_{2}\right)$ such that

$$
f_{2}\left(Z_{2}\right)=W_{2}^{T} S_{2}\left(Z_{2}\right)+\delta_{2}\left(Z_{2}\right)
$$

with $\delta_{2}(Z)$ being the approximation error and satisfying $\left|\delta_{2}\right| \leq \varepsilon_{2}$. Consequently, a simple method computing produces the following inequality:

$$
z_{2} f_{2}=z_{2}\left(W_{2}^{T} S_{2}+\delta_{2}\right) \leq \frac{1}{2 l_{2}^{2}} z_{2}^{2}\left\|W_{2}\right\|^{2} S_{2}^{2}+\frac{1}{2} l_{2}^{2}+\frac{1}{2} z_{2}^{2}+\frac{1}{2} \varepsilon_{2}^{2}
$$

It follows immediately from substituting (3.8) into (3.5) that

$$
\begin{aligned}
\dot{V}_{2} \leq & -\sum_{i=1}^{2} k_{i} z_{i}^{2}+a_{2} z_{1} x_{2} x_{3}+z_{1}(\widehat{B}-B) x_{1}+z_{1}\left(\widehat{T}_{L}-T_{L}\right) \\
& +z_{1}(\widehat{J}-J) \dot{x}_{d}+\frac{1}{2 l_{2}^{2}} z_{2}^{2}\left\|W_{2}\right\|^{2} S_{2}^{2}+\frac{1}{2} l_{2}^{2}+\frac{1}{2} z_{2}^{2}+\frac{1}{2} \varepsilon_{2}^{2}+z_{2} b_{4} u_{q} .
\end{aligned}
$$


The control input $u_{q}$ is designed as

$$
u_{q}=\frac{1}{b_{4}}\left(-k_{2} z_{2}-\frac{1}{2} z_{2}-\frac{1}{2 l_{2}^{2}} z_{2} \widehat{\theta} S_{2}^{2}\right),
$$

where $\widehat{\theta}$ is the estimation of the unknown constant $\theta$ which will be specified later. Using equality (3.10), the derivative of $V_{2}$ becomes as

$$
\begin{aligned}
\dot{V}_{2} \leq & -\sum_{i=1}^{2} k_{i} z_{i}^{2}+a_{2} z_{1} x_{2} x_{3}+z_{1}(\widehat{B}-B) x_{1}+z_{1}\left(\widehat{T}_{L}-T_{L}\right) \\
& +z_{1}(\widehat{J}-J) \dot{x}_{d}+\frac{1}{2 l_{2}^{2}} z_{2}^{2}\left(\left\|W_{2}\right\|^{2}-\widehat{\theta}\right) S_{2}^{2}+\frac{1}{2} l_{2}^{2}+\frac{1}{2} \varepsilon_{2}^{2} .
\end{aligned}
$$

Step 3. At this step, we will construct the control law $u_{d}$. To this end, define $z_{3}=x_{3}$ and choose the following Lyapunov function candidate as $V_{3}=V_{2}+(1 / 2) z_{3}^{2}$. Then the derivative of $V_{3}$ is given by

$$
\begin{aligned}
\dot{V}_{3}= & \dot{V}_{2}+z_{3} \dot{z}_{3} \leq-\sum_{i=1}^{2} k_{i} z_{i}^{2}+a_{2} z_{1} x_{2} x_{3}+z_{1}(\widehat{B}-B) x_{1}+z_{1}\left(\widehat{T}_{L}-T_{L}\right) \\
& +z_{1}(\widehat{J}-J) \dot{x}_{d}+\frac{1}{2 l_{2}^{2}} z_{2}^{2}\left(\left\|W_{2}\right\|^{2}-\widehat{\theta}\right) S_{2}^{2}+\frac{1}{2} l_{2}^{2}+\frac{1}{2} \varepsilon_{2}^{2}+z_{3}\left(f_{3}\left(Z_{3}\right)+c_{3} u_{d}\right)
\end{aligned}
$$

where $f_{3}\left(Z_{3}\right)=a_{2} z_{1} x_{2}+c_{1} x_{3}+c_{2} x_{1} x_{2}$ and $Z_{3}=\left[x_{1}, x_{2}, x_{3}, x_{d}\right]^{T}$. Similarly, by Lemma 2.3 the fuzzy logic system $W_{3}^{T} S_{3}\left(Z_{3}\right)$ is utilized to approximate the nonlinear function $f_{3}$ such that for given $\varepsilon_{3}>0$,

$$
z_{3} f_{3} \leq \frac{1}{2 l_{3}^{2}} z_{3}^{2}\left\|W_{3}\right\|^{2} S_{3}^{2}+\frac{1}{2} l_{3}^{2}+\frac{1}{2} z_{3}^{2}+\frac{1}{2} \varepsilon_{3}^{2} .
$$

Substituting (3.13) into (3.12) gives

$$
\begin{aligned}
\dot{V}_{3}= & \dot{V}_{2}+z_{3} \dot{z}_{3} \leq-\sum_{i=1}^{2} k_{i} z_{i}^{2}+\frac{1}{2 l_{2}^{2}} z_{2}^{2}\left(\left\|W_{2}\right\|^{2}-\widehat{\theta}\right) S_{2}^{2}+\sum_{i=2}^{3} \frac{1}{2}\left(l_{i}^{2}+\varepsilon_{i}^{2}\right) \\
& +z_{1}(\widehat{B}-B) x_{1}+z_{1}\left(\widehat{T}_{\mathrm{L}}-T_{L}\right)+z_{1}(\widehat{J}-J) \dot{x}_{d}+\frac{1}{2 l_{3}^{2}} z_{3}^{2}\left\|W_{3}\right\|^{2} S_{3}^{2}+\frac{1}{2} z_{3}^{2}+c_{3} z_{3} u_{d}
\end{aligned}
$$

Now design $u_{d}$ as

$$
u_{d}=\frac{-1}{c_{3}}\left(k_{3} z_{3}+\frac{1}{2} z_{3}+\frac{1}{2 l_{3}^{2}} z_{3} \widehat{\theta} S_{3}^{2}\right) .
$$


Then, define $\theta=\max \left\{\left\|W_{2}\right\|^{2},\left\|W_{3}\right\|^{2}\right\}$. Then, combining (3.14) with (3.15) results in

$$
\begin{aligned}
\dot{V}_{4} \leq & -\sum_{i=1}^{3} k_{i} z_{i}^{2}+\sum_{i=2}^{3} \frac{1}{2}\left(l_{i}^{2}+\varepsilon_{i}^{2}\right)+z_{1}(\widehat{B}-B) x_{1}+z_{1}\left(\widehat{T}_{L}-T_{L}\right) \\
& +z_{1}(\widehat{J}-J) \dot{x}_{d}+\sum_{i=2}^{3} \frac{1}{2 l_{i}^{2}} z_{i}^{2}\left(\left\|W_{i}\right\|^{2}-\widehat{\theta}\right) S_{i}^{T}\left(Z_{i}\right) S_{i}\left(Z_{i}\right) \\
\leq & -\sum_{i=1}^{3} k_{i} z_{i}^{2}+\sum_{i=2}^{3} \frac{1}{2}\left(l_{i}^{2}+\varepsilon_{i}^{2}\right)+z_{1}(\widehat{B}-B) x_{1}+z_{1}\left(\widehat{T}_{L}-T_{L}\right) \\
& +z_{1}(\widehat{J}-J) \dot{x}_{d}+\sum_{i=2}^{3} \frac{1}{2 l_{i}^{2}} z_{i}^{2} S_{i}^{T}\left(Z_{i}\right) S_{i}\left(Z_{i}\right)(\theta-\widehat{\theta}) .
\end{aligned}
$$

At the present stage, to estimate the unknown constants $B, T_{L}, J$, and $\theta$, define the adaptive variables as follows:

$$
\begin{aligned}
\widetilde{T}_{L} & =\widehat{T}_{L}-T_{L}, \\
\widetilde{B} & =\widehat{B}-B, \\
\widetilde{J} & =\widehat{J}-J, \\
\tilde{\theta} & =\widehat{\theta}-\theta .
\end{aligned}
$$

In order to determine the corresponding adaptation laws, choose the following Lyapunov function candidate:

$$
V=V_{3}+\frac{1}{2 r_{1}} \widetilde{T}_{L}^{2}+\frac{1}{2 r_{2}} \widetilde{B}^{2}+\frac{1}{2 r_{3}} \widetilde{J}^{2}+\frac{1}{2 r_{4}} \widetilde{\theta}^{2}
$$

where $r_{i}, i=1,2,3,4$ are positive constant. By differentiating $V$ and taking (3.16)-(3.18) into account, one has

$$
\begin{aligned}
\dot{V} \leq & -\sum_{i=1}^{3} k_{i} z_{i}^{2}+\sum_{i=2}^{3} \frac{1}{2}\left(l_{i}^{2}+\varepsilon_{i}^{2}\right)+z_{1} \widetilde{B} x_{1}+z_{1} \widetilde{T}_{L} \\
& +z_{1} \tilde{J}_{d}-\sum_{i=2}^{3} \frac{1}{2 l_{i}^{2}} z_{i}^{2} \tilde{\theta} S_{i}^{T}\left(Z_{i}\right) S_{i}\left(Z_{i}\right)+\frac{1}{r_{1}} \widetilde{T}_{L} \widehat{T}_{L}+\frac{1}{r_{2}} \widetilde{B} \widehat{B}+\frac{1}{r_{3}} \tilde{J} \widehat{J}+\frac{1}{r_{4}} \tilde{\theta} \widehat{\theta} \\
= & -\sum_{i=1}^{3} k_{i} z_{i}^{2}+\sum_{i=2}^{3} \frac{1}{2}\left(l_{i}^{2}+\varepsilon_{i}^{2}\right)+\frac{1}{r_{1}} \widetilde{T}_{L}\left(r_{1} z_{1}+\dot{\widehat{T}}_{L}\right) \\
& +\frac{1}{r_{2}} \widetilde{B}\left(r_{2} z_{1} x_{1}+\dot{\hat{B}}\right)+\frac{1}{r_{3}} \tilde{J}\left(r_{3} z_{1} \dot{x}_{d}+\dot{\hat{J}}\right)+\frac{1}{r_{4}} \tilde{\theta}\left[-\sum_{i=2}^{3} \frac{r_{4}}{2 l_{i}^{2}} z_{i}^{2} S_{i}^{T}\left(Z_{i}\right) S_{i}\left(Z_{i}\right)+\dot{\hat{\theta}}\right] .
\end{aligned}
$$


According to (3.19), the corresponding adaptive laws are chosen as follows:

$$
\begin{aligned}
\dot{\hat{T}}_{L} & =-r_{1} z_{1}-m_{1} \widetilde{T}_{L}, \\
\dot{\hat{B}} & =-r_{2} z_{1} x_{1}-m_{2} \widehat{B}, \\
\dot{\hat{J}} & =-r_{3} z_{1} \dot{x}_{d}-m_{3} \widehat{J}, \\
\dot{\hat{\theta}} & =\sum_{i=2}^{3} \frac{r_{4}}{2 l_{i}^{2}} z_{i}^{2} S_{i}^{T}\left(Z_{i}\right) S_{i}\left(Z_{i}\right)-m_{4} \widehat{\theta},
\end{aligned}
$$

where $m_{i}$ for $i=1,2,3,4$ and $l_{i}$ for $i=2,3$ are positive constant.

\section{Stability Analysis}

In this section, the stability analysis of the resulting closed-loop system will be addressed. Substituting (3.20) into (3.19) yields

$$
\dot{V} \leq-\sum_{i=1}^{3} k_{i} z_{i}^{2}+\sum_{i=2}^{3} \frac{1}{2}\left(l_{i}^{2}+\varepsilon_{i}^{2}\right)-\frac{m_{1}}{r_{1}} \widetilde{T}_{L} \widehat{T}-\frac{m_{2}}{r_{2}} \widetilde{B} \widehat{B}-\frac{m_{3}}{r_{3}} \widetilde{J} \widehat{J}-\frac{m_{4}}{r_{4}} \tilde{\theta} \widehat{\theta}
$$

For the term $-\widetilde{T}_{L} \widehat{T}$, one has

$$
-\widetilde{T}_{L} \widehat{T}_{L}=-\widetilde{T}_{L}\left(\widetilde{T}_{L}+T_{L}\right) \leq-\frac{1}{2} \widetilde{T}_{L}^{2}+\frac{1}{2} T_{L}^{2}
$$

Similarly, we have

$$
\begin{aligned}
& -\widetilde{B} \widehat{B} \leq-\frac{1}{2} \widetilde{B}^{2}+\frac{1}{2} B^{2}, \\
& -\tilde{J} \widehat{J} \leq-\frac{1}{2} \widetilde{J}^{2}+\frac{1}{2} J^{2} \\
& -\tilde{\theta} \widehat{\theta} \leq-\frac{1}{2} \widetilde{\theta}^{2}+\frac{1}{2} \theta^{2} .
\end{aligned}
$$

Consequently, by using these inequalities, (4.1) can be rewritten in the following form:

$$
\begin{aligned}
\dot{V} \leq & -\sum_{i=1}^{3} k_{i} z_{i}^{2}-\frac{m_{1}}{2 r_{1}} \widetilde{T}_{L}^{2}-\frac{m_{2}}{2 r_{2}} \widetilde{B}^{2}-\frac{m_{3}}{2 r_{3}} \widetilde{J}^{2}-\frac{m_{4}}{2 r_{4}} \widetilde{\theta}^{2} \\
& +\sum_{i=2}^{3} \frac{1}{2}\left(l_{i}^{2}+\varepsilon_{i}^{2}\right)+\frac{m_{1}}{2 r_{1}} T_{L}^{2}+\frac{m_{2}}{2 r_{2}} B^{2}+\frac{m_{3}}{2 r_{3}} J^{2}+\frac{m_{4}}{2 r_{4}} \theta^{2} \\
\leq & -a_{0} V+b_{0},
\end{aligned}
$$


where $a_{0}=\min \left\{2 k_{1} / J, 2 k_{2}, 2 k_{3}, m_{1}, m_{2}, m_{3}, m_{4}\right\}$ and $b_{0}=\sum_{i=2}^{3}(1 / 2)\left(l_{i}^{2}+\varepsilon_{i}^{2}\right)+\left(m_{1} / 2 r_{1}\right) T_{L}^{2}+$ $\left(m_{2} / 2 r_{2}\right) B^{2}+\left(m_{3} / 2 r_{3}\right) J^{2}+\left(m_{4} / 2 r_{4}\right) \theta^{2}$. Furthermore, (4.4) implies that

$$
V(t) \leq\left(V\left(t_{0}\right)-\frac{b_{0}}{a_{0}}\right) e^{-a_{0}\left(t-t_{0}\right)}+\frac{b_{0}}{a_{0}} \leq V\left(t_{0}\right)+\frac{b_{0}}{a_{0}}, \quad \forall t \geqslant t_{0} .
$$

As a result, all $z_{i}(i=1,2,3), \widetilde{T}_{L}, \widetilde{B}, \tilde{J}$ and $\tilde{\theta}$ belong to the compact set

$$
\Omega=\left\{\left(z_{i}, \tilde{T}_{L}, \tilde{B}, \tilde{J}, \tilde{\theta}\right) \mid V \leq V\left(t_{0}\right)+\frac{b_{0}}{a_{0}}, \forall t \geqslant t_{0}\right\} .
$$

Namely, all the signals in the closed-loop system are bounded. Especially, from (4.5) we have

$$
\lim _{t \rightarrow \infty} z_{1}^{2} \leq \frac{2 b_{0}}{a_{0}}
$$

From the definitions of $a_{0}$ and $b_{0}$, it is clear that to get a small tracking error we can take $r_{i}$ large and $l_{i}$ and $\varepsilon_{i}$ small enough after giving the parameters $k_{i}$ and $m_{i}$.

\section{Simulation}

To illustrate the effectiveness of the proposed results, the simulation will be done for the PMSM motor with the parameters:

$$
\begin{aligned}
J=0.00379 \mathrm{Kgm}^{2}, \quad R_{s}=0.68 \Omega, & L_{d}=0.00315 \mathrm{H}, \\
L_{q}=0.00285 \mathrm{H}, \quad B=0.001158 \mathrm{Nm} /(\mathrm{rad} / \mathrm{s}), & \Phi=0.1245 \mathrm{~Wb}, \quad n_{p}=3 .
\end{aligned}
$$

Then, the proposed adaptive fuzzy controllers are used to control this PMSM motor. Given the reference signal is $x_{d}=30$ and the control parameters are chosen as follows:

$$
\begin{gathered}
k_{1}=2.5, \quad k_{2}=k_{3}=50, \quad r_{1}=r_{2}=r_{3}=r_{4}=2.5, \\
m_{1}=m_{2}=m_{3}=m_{4}=0.0005, \quad l_{2}=l_{3}=5 .
\end{gathered}
$$




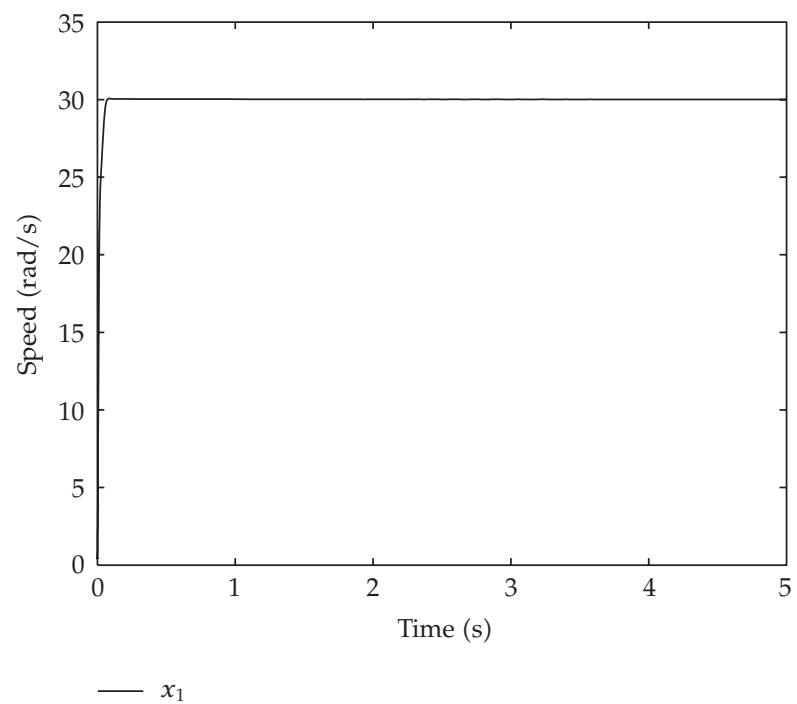

Figure 1: The curve of the rotor speed $x_{1}$.

The fuzzy membership functions are chosen as

$$
\begin{aligned}
& \mu_{F_{i}^{1}}=\exp \left[\frac{-(x+5)^{2}}{2}\right], \quad \mu_{F_{i}^{2}}=\exp \left[\frac{-(x+4)^{2}}{2}\right], \\
& \mu_{F_{i}^{3}}=\exp \left[\frac{-(x+3)^{2}}{2}\right], \quad \mu_{F_{i}^{4}}=\exp \left[\frac{-(x+2)^{2}}{2}\right], \\
& \mu_{F_{i}^{5}}=\exp \left[\frac{-(x+1)^{2}}{2}\right], \quad \mu_{F_{i}^{6}}=\exp \left[\frac{-(x-0)^{2}}{2}\right] \\
& \mu_{F_{i}^{7}}=\exp \left[\frac{-(x-1)^{2}}{2}\right], \quad \mu_{F_{i}^{8}}=\exp \left[\frac{-(x-2)^{2}}{2}\right] \\
& \mu_{F_{i}^{9}}=\exp \left[\frac{-(x-3)^{2}}{2}\right], \quad \mu_{F_{i}^{10}}=\exp \left[\frac{-(x-4)^{2}}{2}\right] \\
& \mu_{F_{i}^{11}}=\exp \left[\frac{-(x-5)^{2}}{2}\right] .
\end{aligned}
$$

The simulation is carried out under the zero initial condition for two cases. In the first case, $T_{L}=1.5$ and in the second case,

$$
T_{L}= \begin{cases}1.5, & 0 \leq t \leq 1 \\ 3, & t \geq 1\end{cases}
$$




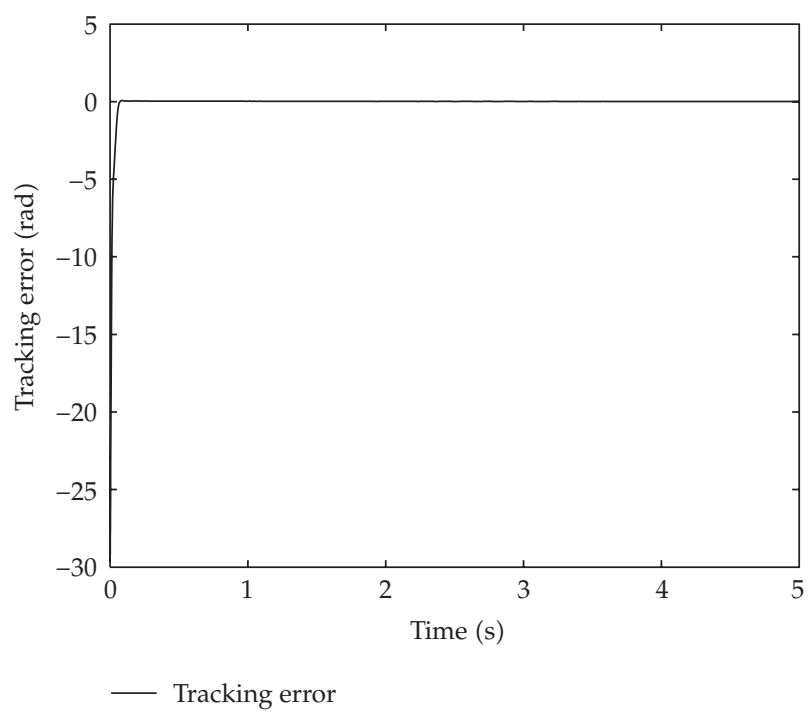

Figure 2: The curve of the speed tracking error.

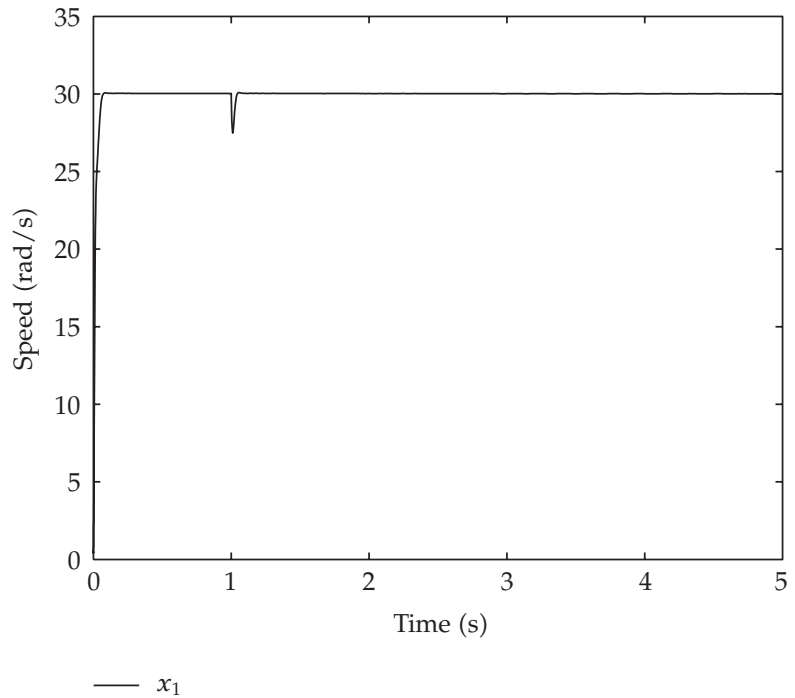

Figure 3: The curve of the rotor position $x_{1}$.

Figures 1 and 2 show the simulation results for case 1, and Figures 3 and 4 show the simulation results for the second case. From these figures, it is seen clearly that the tracking performance has been achieved very well. This means that the proposed controller can track the reference signal satisfactorily even under parameter uncertainties and load torque disturbance.

\section{Conclusion}

Based on adaptive fuzzy control approach and backstepping technique, an adaptive fuzzy control scheme is proposed to control a permanent magnet synchronous motor. The proposed 


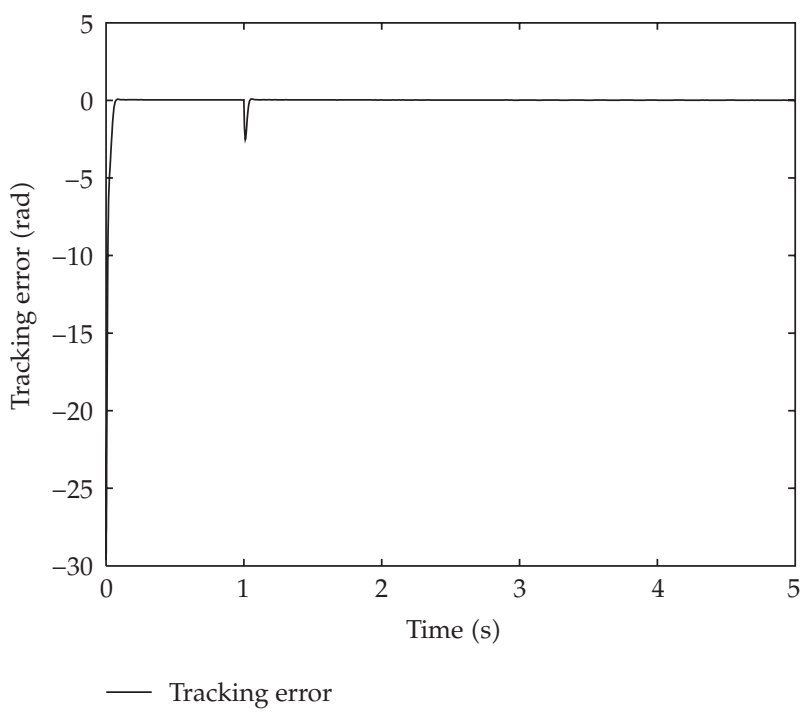

Figure 4: The curve of the speed tracking error.

controllers guarantee that the tracking error converges to a small neighborhood of the origin and all the closed-loop signals are bounded. Simulation results illustrate the effectiveness of the presented method.

\section{Appendix}

\section{The Controller of PMSM Based on Conventional Backstepping}

The controller of PMSM based on conventional backstepping technique is briefly reviewed here. The detailed design procedure is in [7]. The controllers based on conventional backstepping are shown as follows:

$$
\begin{aligned}
& u_{q}=\frac{1}{b_{4}}\left(-k_{2} z_{2}+b_{4}\left(\frac{\dot{\hat{J}}}{a_{1}}\left(-k_{1} z_{1}+\widehat{F} x_{1}+\widehat{\Gamma}+\dot{x}_{d}\right)+\frac{\hat{J}}{a_{1}}\left(\dot{\hat{F}} x_{1}+\dot{\hat{\Gamma}}+\ddot{x}_{d}+k_{1} \dot{x}_{d}\right)\right.\right. \\
& \left.\left.+\left(\widehat{F}-k_{1}\right)\left(x_{2}+\frac{a_{2}}{a_{1}} x_{2} x_{3}\right)-\frac{\widehat{J}}{a_{1}}\left(\widehat{F}-k_{1}\right)\left(\widehat{F} x_{1}+\widehat{\Gamma}\right)-\left(b_{1} x_{2}+b_{2} x_{1} x_{3}+b_{3} x_{1}\right)\right)\right), \\
& u_{d}=\frac{1}{c_{3}}\left(-k_{3} z_{3}-c_{1} x_{3}-c_{2} x_{1} x_{2}-\frac{a_{2}}{\widehat{J}} c_{3} z_{1} x_{2}\right) .
\end{aligned}
$$

Comparing the presented method with the above controller designed via conventional backstepping, it can be seen that the proposed controllers based on adaptive fuzzy backstepping have a very simple structure. And the simulation results illustrate the effectiveness of the presented method in this paper. 


\section{Acknowledgments}

This work is partially supported by the Natural Science Foundation of China (60674055, 60774027, 60444047), the National 863 High Technology Plan of China (2007AA11Z247), the State Key Laboratory of Rail Traffic Control and Safety (Beijing Jiaotong University) (RCS2008ZZ004, SKL2007K006), the Shan Dong Province Key Laboratory of Industrial Control Technique, and Shandong Province Domestic Visitor Foundation (2007001).

\section{References}

[1] F.-J. Lin and S.-L. Chiu, "Adaptive fuzzy sliding-mode control for PM synchronous servo motor drives," IEE Proceedings: Control Theory and Applications, vol. 145, no. 1, pp. 63-72, 1998.

[2] S. C. Tong and H. H. Li, "Fuzzy adaptive sliding model control for mimo nonlinear systems," IEEE Transactions on Fuzzy Systems, vol. 11, no. 3, pp. 354-360, 2003.

[3] A. Isidori, Nonlinear Control Systems, Communications and Control Engineering Series, Springer, Berlin, Germany, 3rd edition, 1995.

[4] H. Lee and M. Tomizuka, "Robust adaptive control using a universal approximator for SISO nonlinear systems," IEEE Transactions on Fuzzy Systems, vol. 8, no. 1, pp. 95-106, 2000.

[5] M. Krstic, I. Kanellakopoulos, and P. Kokotovic, Nonlinear and Adaptive Control Design, John Wiley \& Sons, New York, NY, USA, 1995.

[6] X. Liu, G. Gu, and K. Zhou, "Robust stabilization of MIMO nonlinear systems by backstepping," Automatica, vol. 35, no. 5, pp. 987-992, 1999.

[7] J.-H. Hu and J.-B. Zou, "Adaptive backstepping control of permanent magnet synchronous motors with parameter uncertainties," Control and Decision, vol. 21, no. 11, pp. 1264-1269, 2006.

[8] L. A. Zadeh, "Fuzzy sets," Information and Computation, vol. 8, pp. 338-353, 1965.

[9] C. Elmas, O. Ustun, and H. H. Sayan, "A neuro-fuzzy controller for speed control of a permanent magnet synchronous motor drive," Expert Systems with Applications, vol. 34, no. 1, pp. 657-664, 2008.

[10] S. Tong and H.-X. Li, "Direct adaptive fuzzy output tracking control of nonlinear systems," Fuzzy Sets and Systems, vol. 128, no. 1, pp. 107-115, 2002.

[11] C. C. Lee, "Fuzzy logic in control systems: fuzzy logic controller-I," IEEE Transactions on Systems, Man, and Cybernetics, vol. 20, no. 2, pp. 404-418, 1990.

[12] C. C. Lee, "Fuzzy logic in control systems: fuzzy logic controller-II," IEEE Transactions on Systems, Man, and Cybernetics, vol. 20, no. 2, pp. 419-435, 1990.

[13] P. Pillay and R. Krishnan, "Modeling of permanent magnet motor drives," IEEE Transactions on Industrial Electronics, vol. 35, no. 4, pp. 537-541, 1988.

[14] W. Leonhard, Control of Electrical Drives, Springer, Berlin Germany, 1985.

[15] L.-X. Wang and J. M. Mendel, "Fuzzy basis functions, universal approximation, and orthogonal leastsquares learning," IEEE Transactions on Neural Networks, vol. 3, no. 5, pp. 807-814, 1992. 


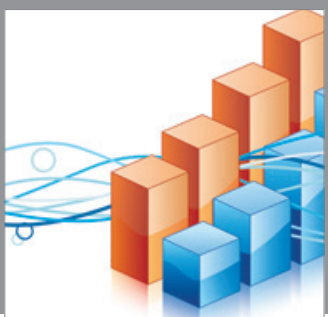

Advances in

Operations Research

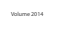

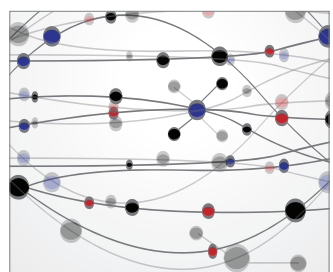

\section{The Scientific} World Journal
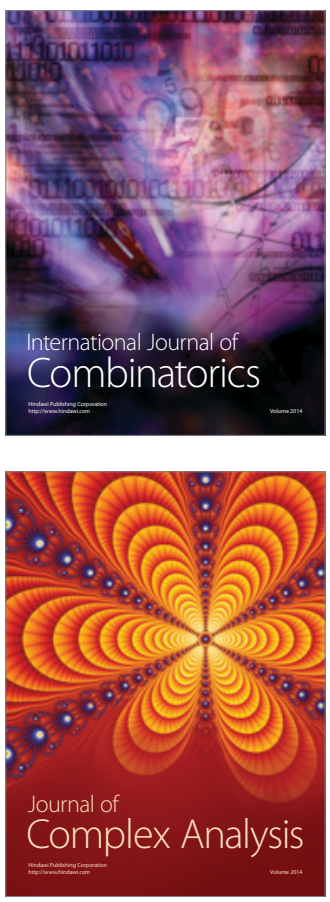

International Journal of

Mathematics and

Mathematical

Sciences
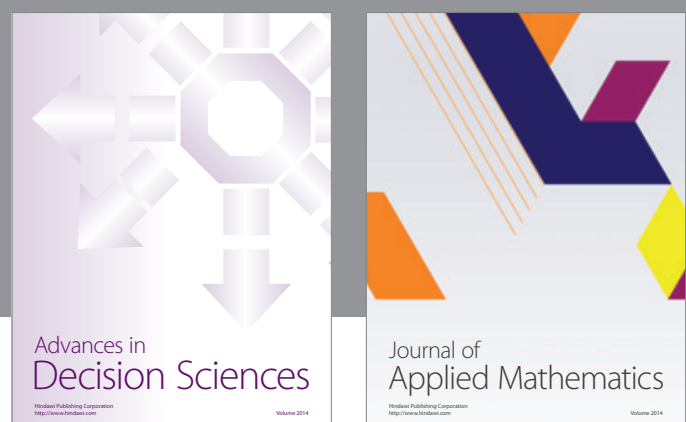

Journal of

Applied Mathematics
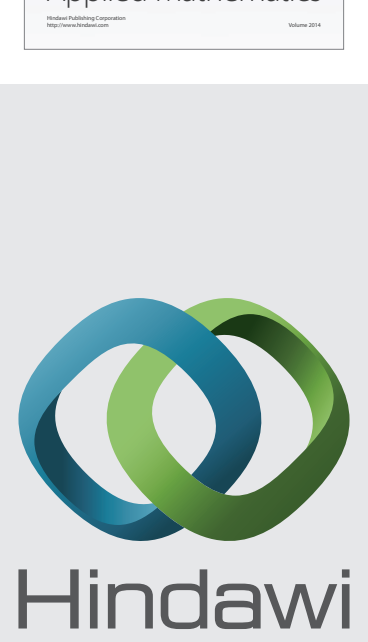

Submit your manuscripts at http://www.hindawi.com
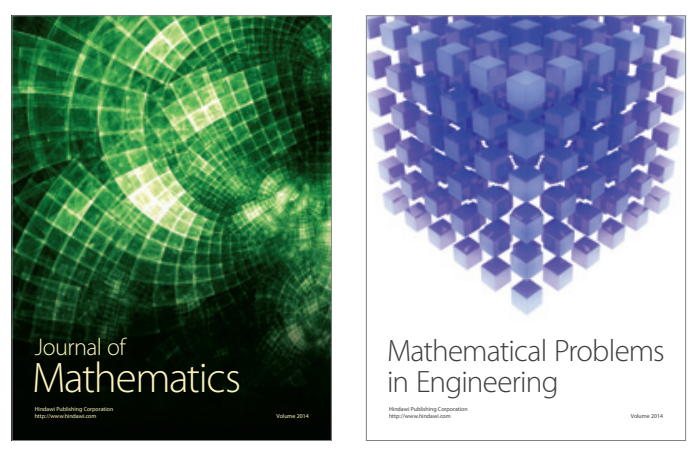

Mathematical Problems in Engineering
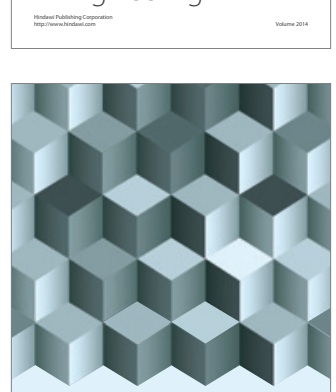

Journal of

Function Spaces
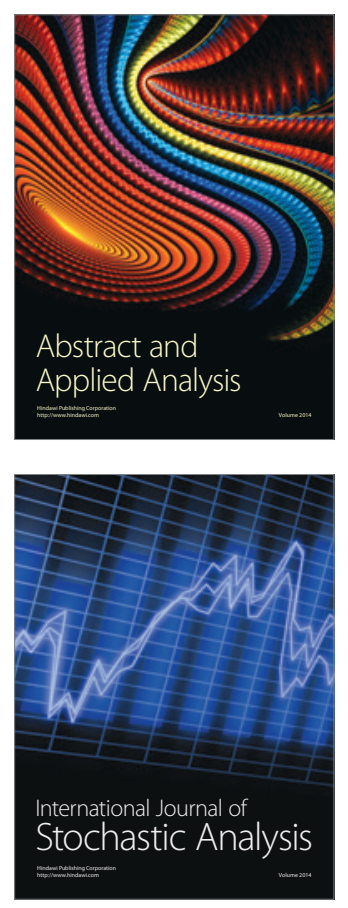

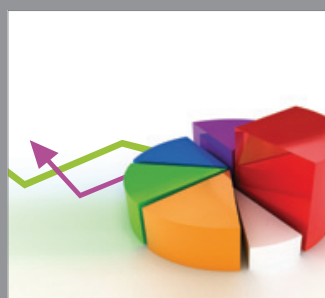

ournal of

Probability and Statistics

Promensencen
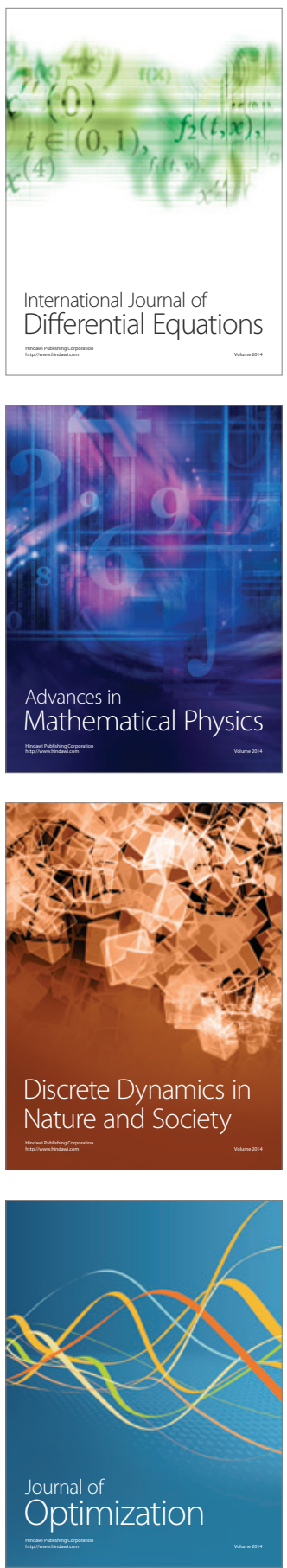